\title{
Pasireotide: successful treatment of a sparsely granulated tumour in a resistant case of acromegaly
}

\author{
W K M G Amarawardena',2, K D Liyanarachchi1,2, J D C Newell-Price ${ }^{1,2}$, R J M Ross', \\ D lacovazzo 3 and $M$ Debono $^{2}$ \\ 1Department of Oncology and Metabolism, University of Sheffield, Sheffield, UK, 2Department of \\ Endocrinology, Royal Hallamshire Hospital, University of Sheffield, Sheffield, UK, and ${ }^{3}$ Centre for \\ Endocrinology, William Harvey Research Institute, Queen Mary University of London, London, UK
}

\author{
Correspondence \\ should be addressed \\ to W K M G Amarawardena \\ Email \\ maheshiamarawardena@ \\ gmail.com
}

\section{Summary}

The granulation pattern of somatotroph adenomas is well known to be associated with differing clinical and biochemical characteristics, and it has been shown that sparsely granulated tumours respond poorly to commonly used somatostatin receptor ligands (SRLs). We report a challenging case of acromegaly with a sparsely granulated tumour resistant to multiple modalities of treatment, ultimately achieving biochemical control with pasireotide. A 26-year-old lady presented with classical features of acromegaly, which was confirmed by an oral glucose tolerance test. Insulin-like growth factor 1 (IGF1) was $1710 \mu \mathrm{g} / \mathrm{L}(103-310 \mu \mathrm{g} / \mathrm{L})$ and mean growth hormone (GH) was $>600 \mathrm{U} / \mathrm{L}$. MRI scan showed a $4 \mathrm{~cm}$ pituitary macroadenoma with suprasellar extension and right-sided cavernous sinus invasion. She underwent trans-sphenoidal pituitary surgery. Histology displayed moderate amounts of sparsely granular eosinophilic cytoplasm, staining only for GH. Postoperative investigations showed uncontrolled disease (IGF1:1474 $\mu \mathrm{g} / \mathrm{L}$, mean GH:228U/L) and residual tumour in the cavernous sinus. She received external beam fractionated radiation. Over the years, she received octreotide LAR (up to $30 \mathrm{mg}$ ), lanreotide (up to $120 \mathrm{mg}$ ) two weekly, cabergoline, pegvisomant and stereotactic radiosurgery to no avail. Only pegvisomant resulted in an element of disease control; however, this had to be stopped due to abnormal liver function tests. Fifteen years after the diagnosis, she was started on pasireotide $40 \mathrm{mg}$ monthly. Within a month, her IGF1 dropped and has remained within the normal range $(103-310 \mu \mathrm{g} / \mathrm{L})$. Pasireotide has been well tolerated, and there has been significant clinical improvement. Somatostatin receptor subtyping revealed a positivity score of two for both sst5 and sst2a subtypes.

\section{Learning points:}

- Age, size of the tumour, GH levels on presentation, histopathological type and the somatostatin receptor status of the tumour in acromegaly should be reviewed in patients who poorly respond to first-generation somatostatin receptor ligands.

- Tumours that respond poorly to first-generation somatostatin receptor ligands, especially sparsely granulated somatotroph adenomas, can respond to pasireotide and treatment should be considered early in the management of resistant tumours.

- Patients with membranous expression of sst5 are likely to be more responsive to pasireotide. 


\section{Background}

Morbidity and mortality rates of uncontrolled acromegaly are much higher than those of the general population. However, adequate biochemical control reverses mortality rates. Currently, medical therapy is becoming an important part of the treatment of patients with acromegaly. Long-acting formulations of octreotide and lanreotide, first-generation somatostatin receptor ligands (SRLs), that mainly bind to receptor sst2, remain the standard medical therapy and play an important role when either surgery is ineffective or as a first-line therapy when surgery is contraindicated. Pasireotide is a somatostatin receptor ligand, acting on receptors sst1, sst2, sst 3 and sst5 and has recently been approved for the treatment of acromegaly. The granulation pattern of somatotroph adenomas is well known to be associated with differing clinical and biochemical characteristics, and it has been shown that sparsely granulated tumours respond poorly to first-generation somatostatin receptor ligands. We report a challenging case of acromegaly due to a sparsely granulated pituitary adenoma, resistant to multiple modalities of treatment administered over several years and ultimately achieving biochemical control with pasireotide.

\section{Case presentation}

A 26-year-old lady presented with tiredness, progressive enlargement of hands and feet, secondary amenorrhoea, gradually worsening headache together with excessive sweating for three years. There was no family history of pituitary tumours, hypercalcaemia or renal stones. Examination revealed typical features of acromegaly. Her blood pressure and visual fields were normal.

\section{Investigations}

The oral glucose tolerance test (OGTT) was performed to confirm the diagnosis of acromegaly (nadir GH: $772 \mathrm{U} / \mathrm{L}$ ). Her IGF-1 level was $1710 \mu \mathrm{g} / \mathrm{L}(103-310 \mu \mathrm{g} / \mathrm{L})$. A growth hormone day curve was performed to assess the disease burden, and it highlighted the presence of severe disease (mean GH >600 U/L). Other anterior pituitary hormone testing showed secondary hypogonadism ( $\mathrm{LH}<0.5 \mathrm{IU} / \mathrm{L}$ (2.4-13 IU/L), FSH <0.37 IU/L (3.5-13 IU/L), oestradiol $<50 \mathrm{pmol} / \mathrm{L}(91.8-854 \mathrm{pmol} / \mathrm{L})$, elevated prolactin of 796 U/L(102-496U/L)) andhypothyroidism(TSH:10.8IU/L $(0.27-4.2 \mathrm{IU} / \mathrm{L})$, free $\mathrm{T} 4$ of $6 \mathrm{pmol} / \mathrm{L}(12-22 \mathrm{pmol} / \mathrm{L}))$. MRI scan showed a $4 \times 2 \times 3 \mathrm{~cm}$ pituitary macro adenoma with suprasellar extension and right-sided cavernous sinus invasion.

\section{Treatment}

The patient underwent trans-sphenoidal surgery of the pituitary tumour in 1999. Histology of the excised specimen revealed moderate amounts of finely (sparsely) granular eosinophilic cytoplasm. Immunohistochemistry was positive for growth hormone but negative for other pituitary hormones. Ki 67 index was $<1 \%$.

Her postoperative IGF1 was $1474 \mu \mathrm{g} / \mathrm{L}$ and mean $\mathrm{GH}$ was $228 \mathrm{U} / \mathrm{L}$, indicating uncontrolled disease. Postoperative MRI of the pituitary revealed residual tumour in the cavernous sinus and close to the optic chiasm. Therefore, external beam fractionated radiation was given five months after surgery.

Octreotide treatment was initiated postoperatively, as $20 \mathrm{mg}$ every 4 weeks. It was then increased stepwise, up to $30 \mathrm{mg}$ every two weeks. The lowest IGF1 achieved was $1065 \mu \mathrm{g} / \mathrm{L}$ indicating partial resistance. Octreotide was stopped in September 2004.

Due to the persistent elevation of biochemical markers over four years following surgery, she was started on pegvisomant, which resulted in reasonable disease control. Her IGF-1 levels came down from above 1000 to $340 \mu \mathrm{g} / \mathrm{L}$. Within a few months of pegvisomant therapy, there was a positive clinical response; however, she developed abnormal liver function tests necessitating discontinuation of the treatment. She was re-challenged with pegvisomant, but in view of a recurrent rise in liver enzymes, treatment was stopped.

Lanreotide was initiated in January 2005 as $120 \mathrm{mg}$ monthly and was soon increased to twice weekly. In April, cabergoline $0.5 \mathrm{mg}$ twice a week was added to lanreotide, but it was stopped in 2006 as it did not have a positive impact on disease control. In 2010, the patient developed diarrhoea and the lanreotide frequency was reduced back to once a month. In 2010, patient had stereotactic radio surgery targeting residual tumour within the cavernous sinuses.

Lanreotide was stopped in 2013 as the patient underwent a clinical trial with antisense, a form of siRNA treatment. Antisense was not able to control the disease and following the trial, she went back to lanreotide treatment with an IGF-1 of $1258 \mu \mathrm{g} / \mathrm{L}$. She was on lanreotide till October 2015. 


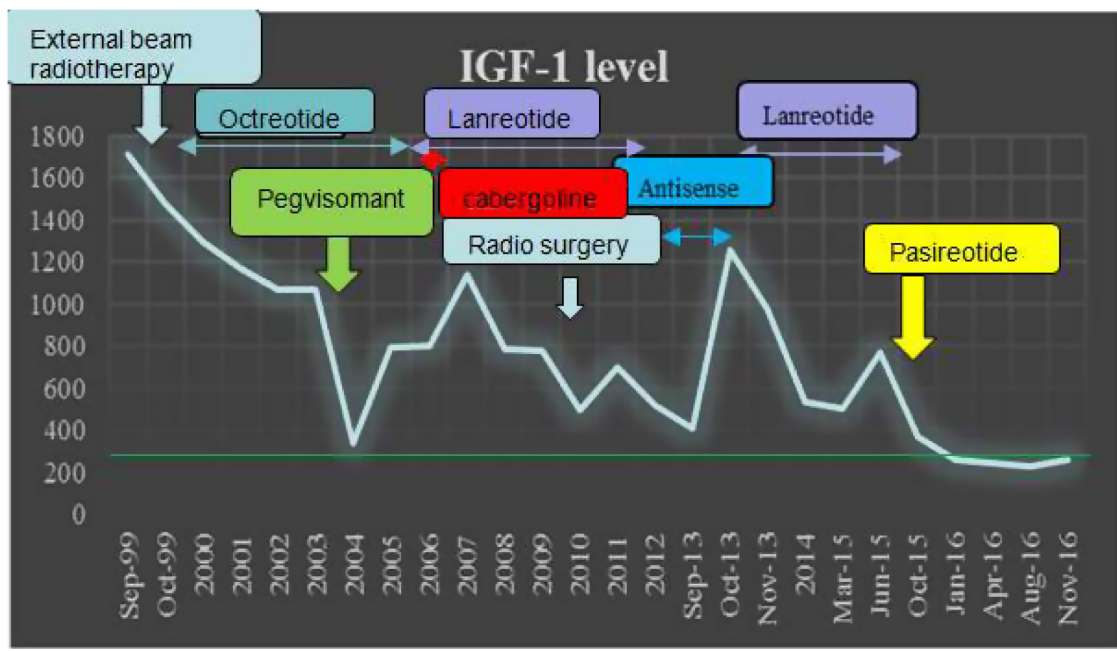

Figure 1

The graph illustrates the relationship between different modalities of clinical therapy with IGF 1 levels.
Over fifteen years, no treatment was able to control the disease burden, and the patient remained highly symptomatic with headaches, fatigue and osteoarthritis. In 2015 , the patient was started on pasireotide $40 \mathrm{mg}$ monthly. Figure 1 shows her clinical therapy and hormone levels.

\section{Outcome and follow-up}

Within a month of starting pasireotide $40 \mathrm{mg}$ monthly, her IGF-1 levels dropped to the normal range and have remained within the normal range for age (103-310 $\mu \mathrm{g} / \mathrm{L})$ over 15 months although her tumour size has not changed. Late effects of radiotherapy may also have contributed to the normalization of $\mathrm{GH}$ and IGF-1. Pasireotide remains well tolerated and has not been complicated by glucose intolerance. Her HBA1c was closely monitored and values ranged from 41 to $44 \mathrm{mmol} / \mathrm{mol}$. It was $42 \mathrm{mmol} / \mathrm{mol}$ prior to starting pasireotide. The patient's symptoms have significantly improved, and she is now back to work. Somatostatin receptor (sst) sub typing revealed positivity for sst5 and sst2a subtypes (Fig. 2).

\section{Discussion}

Despite availability of multiple treatment modalities, acromegaly sometimes is a challenging condition to treat, and we report a patient whose disease was only controlled with pasireotide. The patient had a sparsely granulated tumour with suprasellar extension and invasion of the cavernous sinus, and these tumours are known to be more difficult to control. Sparsely granulated somatotroph adenomas are commoner among young females, are generally larger than densely granulated somatotroph adenomas, less responsive to somatostatin receptor ligands $(1,2)$ and more frequently reported to have suprasellar extension and cavernous sinus infiltration (3).

A number of factors in our patient are associated with increased resistance to treatment of acromegaly. Preoperative GH levels are an important predictor of remission in acromegaly (4). Extremely high disease burden is indicated by clinical findings, biochemistry and imaging and could have contributed largely to the poor response to initial treatment in our patient.

The response to somatostatin receptor ligands depends on the expression of the somatostatin receptor subtype (5). Pasireotide is a somatostatin receptor ligand recently
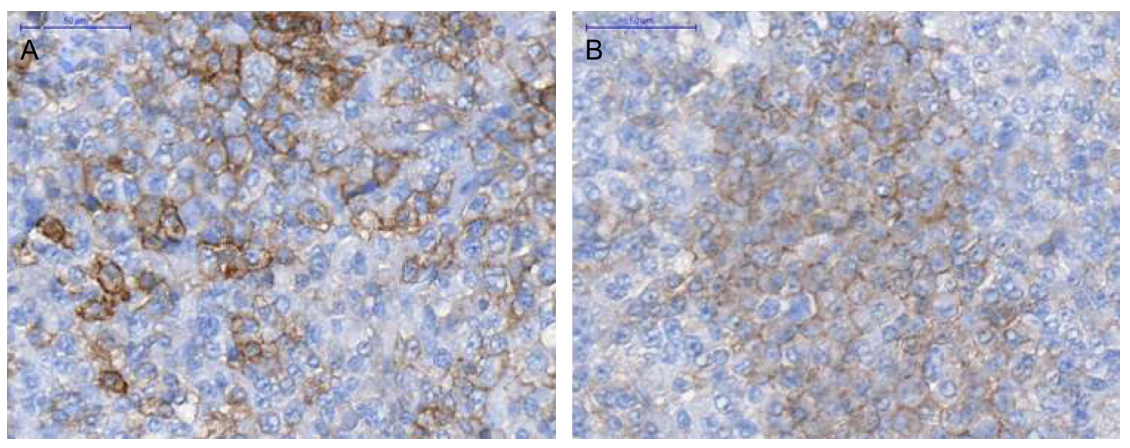

\section{Figure 2}

(A) Immunohistochemistry for sst2a showing membranous expression of sst2a (score 2 according to scoring system proposed by Volante et al. (9)). The staining was performed using the monoclonal UMB-1 (Abcam) on an automated Ventana system. (B) Immunohistochemistry for sst5 showing membranous expression of sst5 (score 2 according to scoring system proposed by Volante et al. (9)). The staining was performed using the monoclonal UMB-4 (Abcam) on an automated Ventana system. 
approved for the treatment of acromegaly. Randomised studies have shown its superiority to octreotide and lanreotide in the treatment of uncontrolled acromegaly (6). In contrast to octreotide and lanreotide, which bind preferentially to somatostatin receptor sst2, pasireotide has a binding affinity for sst5 that is approximately 39- and 106-fold higher and a lower binding affinity for sst2 (approximately 0.4- and 0.5-times lower) (7). A small retrospective study of eleven patients resistant to first-generation SRLs were treated with pasireotide, and it was found that the expression of sst5 could predict the responsiveness to treatment (8). The study also showed that sparsely granulated somatotroph adenomas responded to pasireotide better than densely granulated tumours. Sparsely granulated tumours had a tendency for a lower sst2a expression compared to densely granulated somatotroph adenomas, but no difference was observed for sst5 expression. The study concluded that this somatostatin receptor profile might explain the better responsiveness of sparsely granulated tumours to pasireotide. Our patient had a sparsely granulated tumour, which stained for both sst2a as well as sst5 using the rabbit monoclonal antibodies (Abcam) on the automated Ventana system. The membranous expression of somatostatin receptor is a prerequisite for the responsiveness to different somatostatin receptor ligands (8). Patients with negative or only cytoplasmic sst5 expression (scores $0-1$ according to the scoring system proposed by Volante et al.) (9) are sometimes not considered responsive to pasireotide as opposed to cases with membranous expression of sst5 (scores 2 or 3 in the same scoring system) according to Volante et al. Our patient shows positive expression for both sst2a and sst5 with a score of 2 (i.e. membranous staining in $<50 \%$ of cells or incomplete membranous staining), explaining the good response to pasireotide.

Studies revealed that the tolerability profile of intramuscular pasireotide is generally similar to that of firstgeneration agents, except for a higher incidence of hyperglycaemia-related adverse events with pasireotide, suggesting the importance of careful monitoring of glycaemic status (10). Pasireotide was well tolerated in our patient, and she has not developed hyperglycaemia for a follow-up period of 15 months.

Pasireotide should be considered early in tumours that respond poorly to first-generation somatostatin receptor ligands, especially sparsely granulated somatotroph adenomas with membranous expression of sst5.
Declaration of interest

The authors declare that there is no conflict of interest that could be perceived as prejudicing the impartiality of the research reported.

\section{Funding}

This case report did not receive any specific grant from any funding agency in the public, commercial or not-for-profit sector.

\section{Patient consent}

Written informed consent was obtained from the patient for publication of the submitted article and accompanying images.

\section{Author contribution statement}

W A and $K \mathrm{~L}$ collected the data and W A, K L, J N-P, R J R, D I and M D wrote and contributed to the manuscript. All authors were involved in the direct care of the patient.

\section{References}

1 Obari A, Sano T, Ohyama K, Kudo E, Qian Z, Yoneda A, Rayhan N, Rahman M \& Yamada S 2008 Clinicopathological features of growth hormone-producing pituitary adenomas: difference among various types defined by cytokeratin distribution pattern including a transitional form. Endocrine Pathology 19 82-91. (doi:10.1007/s12022-008-9029-z)

2 Bakhtiar Y, Hirano H, Arita K, Yunoue S, Fujio S, Tominaga A, Sakoguchi T, Sugiyama K, Kurisu K, Takano Y, et al. 2010 Relationship between cytokeratin staining patterns and clinico-pathological features in somatotropinomae. European Journal of Endocrinology 163 531-539. (doi:10.1530/EJE-10-0586)

3 Mazal PR, Czech T, Sedivy R, Aichholzer M, Wanschitz J, Klupp N \& Budka H 2001 Prognostic relevance of intracytoplasmic cytokeratin pattern, hormone expression profile, and cell proliferation in pituitary adenomas of akromegalic patients. Clinical Neuropathology 20 163-171.

4 Roelfsema F, Biermasz N \& Pereira A 2011 Clinical factors involved in the recurrence of pituitary adenomas after surgical remission: a structured review and meta-analysis. Pituitary 15 71-83. (doi:10.1007/ s11102-011-0347-7)

5 Gatto F, Feelders RA, Pas R, Kros JM, Waaijers M, Sprij-Mooij D, Neggers SJCMM, Lelij AJ, Minuto F, Steven W, et al. 2013 Immunoreactivity score using an anti-sst2A receptor monoclona antibody strongly predicts the biochemical response to adjuvant treatment with somatostatin analogs in acromegaly. Journal of Clinical Endocrinology and Metabolism 98 E66-E71. (doi:10.1210/jc.2012-2609)

6 Gadelha M, Bronstein M, Brue T, Coculescu M, Fleseriu M, Guitelman M, Pronin V, Raverot G, Shimon I, Lievre KK, et al. 2014 Pasireotide versus continued treatment with octreotide or lanreotide in patients with inadequately controlled acromegaly (PAOLA): a randomised, phase 3 trial. Lancet Diabetes and Endocrinology 2 875-884. (doi:10.1016/S2213-8587(14)70169-X)

7 Kiseljak-Vassiliades K, Shafi S, Kerr J, Phang T, Kleinschmidt-DeMasters B \& Wierman M 2012 Clinical implications of growth hormone-secreting tumor subtypes. Endocrine 42 18-28. (doi:10.1007/s12020-012-9660-9)

8 Iacovazzo D, Carlsen E, Lugli F, Chiloiro S, Piacentini S, Bianchi A, Giampietro A, Mormando M, Clear AJ, Doglietto F, et al. 2015 Factors 
Endocrinology,

Diabetes \& Metabolism

CASE REPORTS
W K M G Amarawardena and others
Pasireotide: in a resistant case of acromegaly
ID: 17-0067; July 2017

DOI: 10.1530/EDM-17-0067 predicting pasireotide responsiveness in somatotroph pituitary adenomas resistant to first-generation somatostatin analogues: an immunohistochemical study. European Journal of Endocrinology $\mathbf{1 7 4}$ 241-250. (doi:10.1530/EJE-15-0832)

9 Volante M, Brizzi MP, Faggiano A, La RosaS, Rapa I, Ferrero A, Mansueto G, Righi L, Garancini S, Capella C, et al. 2007 Somatostatin receptor type 2A immunohistochemistry in neuroendocrine tumors: a proposal of scoring system correlated with somatostatin receptor scintigraphy. Modern Pathology 20 1172-1182 (doi:10.1038/modpathol.3800954)

10 Wildemberg LE \& Gadelha MR 2016 Pasireotide for the treatment of acromegaly. Expert Opinion on Pharmacotherapy 17 579-588. (doi:10.1517/ 14656566.2016.1146688)

Received in final form 13 June 2017

Accepted 22 June 2017 